\title{
JD17
}

\section{Atomic Data for X-Ray Astronomy}

\author{
Chairperson: A.K. Pradhan
}

Editors: A.K. Pradhan (Chief-Editor), Sultana Nahar, and P.L. Smith 


\title{
IAU XXV JD17: Atomic Data For X-Ray Astronomy
}

\author{
Anil K. Pradhan and Sultana N. Nahar \\ Department of Astronomy, The Ohio State University, Columbus, $\mathrm{OH}$ \\ 43210, USA \\ Peter L. Smith \\ Harvard Smithsonian Center for Astrophysics, Cambridge, MA 02138, \\ USA
}

\begin{abstract}
The Joint Discussion on Atomic Data For X-ray Astronomy addressed all aspects of X-ray astronomy: (I) New Observations, (II) Atomic Theory, (III) Laboratory Measurements (IV) Numerical Simulations, and (V) Databases. There were 22 invited presentations and approximately 40 contributed papers. Following papers are brief summaries of the proceedings.

A detailed description of the aims and contents of JD17 are given at: www.astronomy.ohio-state.edu/ pradhan/Iau/iau.html. The SOC consisted of: N. Brickhouse (USA), H. Hasan (USA), J. Houck (USA), A. Fabian (UK), J. Kaastra (Netherlands), F. Keenan (UK), S. Kahn (USA), T. Kallman (USA), H. Netzer (Israel), A.K. Pradhan (USA, Chair), P.L. Smith (USA), K.Yamashita (Japan).
\end{abstract}

Acknowledgments. The SOC would like to thank the Executive Committee and the Secretariat of the IAU for approving the JD, local arrangements in Sydney, for several travel grants to invited speakers. The chair (AKP) would especially like to thank Dr. Hans Rickman for great and prompt assistance in all of these. 